\title{
Evolución en la calidad de la voz en pacientes disfónicos del Hospital de La Serena tratados con terapia vocal
}

\author{
Changes in the quality of voice in dysphonic patients in the \\ La Serena Hospital Treated with vocal therapy
}

\author{
Carolina Martínez S1.
}

\begin{abstract}
RESUMEN
Introducción: La terapia fonoaudiológica de la disfonía contempla un conjunto de recursos subjetivos cuya eficacia debe ser cuantificada.

Objetivo: En el presente trabajo se pretende cuantificar objetivamente la evolución en la calidad de la voz de pacientes disfónicos usando como referencia los índices de perturbación de la frecuencia fundamental (Jitter) e intensidad (Shimmer), y determinar si la evolución es coherente con la evaluación subjetiva del fonoaudiólogo.

Material y método: Se seleccionaron 27 pacientes disfónicos que completaron la terapia vocal entre 2009 y 2011 y que corresponden al 38\% de las consultas fonoaudiológicas por disfonía, en el Hospital de La Serena. Cada paciente, además de la evaluación subjetiva, contó con una aplicación de análisis acústico inicial y final con el programa PRAAT. Se contrastaron los resultados aplicando análisis estadístico con el software SPSS.

Resultados: Los resultados muestran que se reducen las perturbaciones de la frecuencia e intensidad registradas con el análisis acústico en la misma proporción que el grado subjetivo de la disfonía y que dichas variaciones son independientes a otras variables como la edad, sexo y tipo de disfonía.

Conclusión: Se destaca la importancia de la intervención fonoaudiológica en el tratamiento de la disfonía y la relevancia de la aplicación del análisis acústico como medio de evaluación y seguimiento objetivo.
\end{abstract}

Palabras clave: Terapia vocal, grado de disfonía, Jitter, Shimmer, análisis acústico.

\begin{abstract}
Introduction: Phonoaudiological dysphonia therapy provides a set of subjective resources whose effectiveness must be quantified.

Aim: In the present work is to objectively quantify changes in voice quality of dysphonic patients using as reference indices disturbance of the fundamental frequency
\end{abstract}

\footnotetext{
1 Fonoaudióloga. Servicio de Otorrinolaringología, Hospital de La Serena.
} 
(Jitter) and intensity (Shimmer), and determine whether the development is consistent with the subjective evaluation phonoaudiologist.

Material and method: 27 patients who completed dysphonic voice therapy between 2009 and 2011, corresponding to 38\% of consultations phonoaudiological dysphonia, at the Hospital of La Serena were selected. Each patient in addition to the subjective evaIuation, had initial and final application of the PRAAT acoustic analysis program. Results using the SPSS statistical analysis software were compared.

Results: The results show that disturbances in the frequency and intensity recorded with acoustic analysis in the same proportion will reduce the perceived degree of hoarseness and that these variations are independent of other variables such as age, sex and type of dysphonia.

Conclusion: The importance of speech therapy intervention in the treatment of dysphonia and the relevance of the application of acoustic analysis as a means of objective evaluation and monitoring is emphasized

Key words: Voice therapy, degree of dysphonia, Jitter, Shimmer, acoustic analysis.

\section{INTRODUCCIÓN}

La voz es un sonido que producido por la laringe y modificado por la acción de otras estructuras y sistemas, permiten no sólo la expresión emocional de los individuos ${ }^{1}$, sino también la expresión a través del habla ya que está a la base de gran parte de los sonidos del lenguaje.

Su producción es un fenómeno complejo ${ }^{1}$ en donde interactúan los sistemas neuromuscular, respiratorio, fonatorio, auditivo, endocrino, resonancial y articulatorio, con la coordinación de los subsistemas neurológicos central y periférico². El resultado de esta interacción proporciona las características acústicas que percibimos en la voz: intensidad, altura tonal y timbre. Cuando alguno de estos sistemas se alteran, los parámetros varían y la voz resultante cambia.

Las alteraciones de voz, llamadas disfonías, se producen cuando su realización natural se ve afectada por alguna dificultad en alguno de los niveles de producción alterando uno o varios de sus parámetros ${ }^{3}$. De esta manera, la disfonía puede constituirse como un síntoma único o bien como parte de un conjunto de síntomas de una determinada enfermedad ${ }^{2}$. La severidad de la disfonía dependerá del grado en que se hayan afectado uno 0 más de los parámetros ${ }^{2}$, y del grado de afectación en la vida de relación y en la vida profesional del paciente ${ }^{1}$.

Dada la complejidad de la naturaleza de la voz y de sus alteraciones, el tratamiento de la disfonía es multidisciplinario, contando principalmente con la acción del otorrinolaringólogo, del fonoaudiólogo, del psicólogo, entre otros.

En este contexto, el fonoaudiólogo es el encargado de valorar la funcionalidad de la voz, lo cual hace principalmente mediante mecanismos subjetivos de evaluación que dependen de la experiencia y de la experticia del profesional. También existen herramientas computacionales que permiten realizar valoraciones objetivas de la producción vocal y que permiten a su vez controlar la evolución de un determinado paciente en tratamiento.

La evaluación subjetiva, por parte del fonoaudiólogo, incluye la elaboración de una anamnesis y la aplicación de un examen funcional. Con el primero se obtienen los antecedentes de identificación del paciente, del problema, y los antecedentes psicológicos. Con el segundo se analizan un conjunto de aspectos de la producción que permiten obtener un perfil de la calidad de la voz. Entre los aspectos evaluados, se cuenta, el nivel tensional, la respiración, la intensidad, la altura tonal, timbre, ataque vocal, presencia 0 ausencia de quiebres tonales, etc. La cuantificación de la calidad de la voz puede realizarse aplicando escalas subjetivas descritas en la literatura 0 aplicando escalas de elaboración propia, de ambas maneras se obtiene una impresión global del rendimiento vocal.

Por otra parte, para realizar una evaluación objetiva se pueden utilizar diversas herramientas computacionales, entre las que se cuentan 
Dr Speech (Tiger Elemetric), SSVA (System for Single Voice Analysis), MDVP (Multi-Dimensional Voice Program), EVA (Evaluation Vocal Assistee), CSL (Computerized Speech Laboratory), PRAAT, VISHACSRE (Computerized Speech Research Environment), MEDIVOZ, etc ${ }^{4}$. Cada uno de estos programas analiza la voz grabada a través de un espectograma desde el cual se obtienen una serie de conceptos y valores que permiten diferenciar voces normales y patológicas ${ }^{5}$. Dentro de los índices obtenidos, los más utilizados en la investigación científica son los indicadores de Jitter y Shimmer. El primero es el índice de perturbación de la frecuencia fundamental (F0) ciclo a ciclo que expresado en porcentaje representa la variabilidad de ésta sin tomar en cuenta los cambios voluntarios de ella ${ }^{6}$. El umbral indicado para la normalidad en el PRAAT es de $1,040 \%{ }^{7}$, en donde los valores superiores a éste se consideran patológicos.

El segundo es el indicador de perturbación de la amplitud cuya medición sirve para cuantificar pequeños lapsos de inestabilidad de la señal vo$\mathrm{cal}^{6} \mathrm{y}$ que al igual que el primero se expresa en porcentaje. El valor de normalidad indicado para el PRAAT es de $3,08 \%{ }^{7}$. Se considera también que los valores superiores a ese promedio son indicadores de patología.

En la clínica fonoaudiológica, ambos indicadores son útiles para indicar objetivamente los cambios que se producen en la voz durante el proceso de rehabilitación de un paciente con disfonía, sin embargo, el estudio de esta variación sigue en desarrollo. Existen trabajos que indican que las perturbaciones no se reducen luego de 14 semanas de intervención, como el de Mackenzi, Millar, Sellars y Deary en $2001^{8}$, mientras que en otras investigaciones se afirma que los valores de Jitter y Shimmer se reducen luego de la terapia, estableciendo la herramienta de evaluación acústica como un indicador de éxito ${ }^{9-11}$.

La intervención fonoaudiológica en el tratamiento de la disfonía está orientada a reeducar el uso de la voz, lo cual implica un proceso de aprendizaje que se da en varias etapas en las cuales el paciente adquiere y pone en práctica el conocimiento de una adecuada técnica vocal ${ }^{12}$. Teniendo en cuenta que el objeto de la intervención carece de forma concreta, visible y palpable, el proceso de aprendizaje contempla herramientas de valoración subjetiva y está basado principalmente en aspectos sutiles en donde las sensaciones propioceptivas son la guía más importante ${ }^{13}$.

La cantidad de sesiones de la terapia de voz depende de la respuesta vocal de cada paciente, del grado y de la causa de la disfonía. Los pacientes con una disfonía de corta evolución pueden requerir de 15 a 20 sesiones, mientras que aquellos con una disfonía de larga data podrán estar en terapia durante 80 a 120 sesiones $^{13}$.

Los pacientes del Servicio de Otorrinolaringología del Hospital de La Serena que llegan derivados por disfonía deben aprender la técnica vocal para reducir el grado de disfonía y durante este proceso se utilizan diversos medios didácticos para facilitar el aprendizaje. Uno de los aspectos más relevantes en la terapia es generar en el paciente mecanismos concientes (siempre subjetivos) que le permitan evaluar en conjunto con el terapeuta los progresos obtenidos. En este sentido, la evaluación objetiva contribuye al seguimiento de su evolución, sin embargo, la descripción de la variabilidad esperada de sus indicadores en el contexto terapéutico ha sido poco descrita.

\section{OBJETIVO}

En el presente trabajo se pretende cuantificar objetivamente la evolución en la calidad de la voz de pacientes disfónicos usando como referencia los índices de perturbación de la frecuencia fundamental (Jitter) e intensidad (Shimmer) obtenidos con el software de análisis acústico PRAAT, y determinar si la evolución es positiva y si ésta es coherente con la evaluación subjetiva del profesional fonoaudiólogo.

\section{MATERIAL Y MÉTODO}

Se seleccionaron 27 pacientes disfónicos del Servicio de Otorrinolaringología del Hospital de La Serena que ingresaron a tratamiento fonoaudiológico entre 2009 y 2011 que equivalen al $38 \%$ del total de las consultas por disfonía en ese período. Los sujetos seleccionados contaron con las siguientes características: 
1. Tener diagnóstico otorrinolaringológico de disfonía.

2. Presentar una evaluación fonoaudiológica subjetiva y objetiva al principio de la terapia y al término de ésta.

3. Haber cumplido con todas las etapas relacionadas con el desarrollo de la técnica vocal.

4. No presentar disfonía de origen psicógeno.

El grupo de pacientes seleccionados estaba constituido por 17 adultos y 10 niños. Las edades de los adultos variaban entre los 18 y los 74 años, mientras que la de los niños variaba entre 8 y 13 años. El grupo de adultos lo conformaban 7 hombres y 10 mujeres y en el grupo infantil eran todos varones.

En el grupo de adultos varones, se registraron 2 pacientes con disfonía músculo tensional, 2 con nódulos cordales bilaterales, 3 con parálisis cordal, 1 presentó además reflujo faringolaríngeo y otro reflujo gastroesofágico. En el caso de las mujeres, 6 registraban diagnóstico de disfonía músculo tensional, 1 nódulos cordales bilaterales y 4 presentaban reflujo faringolaríngeo como antecedente. En el caso de los niños, 9 presentaban diagnóstico de nódulos cordales bilaterales y 1 paciente tenía el diagnóstico de disfonía espástica, 1 paciente registró reflujo gastroesofágico y 2 presentaban asma bronquial. Cabe señalar que sólo en el grupo de mujeres se observó además antecedentes de depresión en 4 de ellas y estrés en 5 .

Cada sujeto fue evaluado individualmente en una sala del Servicio de ORL del Hospital de La Serena no insonorizada, pero silenciosa. Se realizaron 2 grabaciones por paciente, una de ellas durante la evaluación inicial y la otra al término del proceso terapéutico. Se utilizó un micrófono cuya frecuencia de respuesta está entre 80 y 16.000 hz. El paciente debía emitir una /a/ sostenida por al menos 3 segundos usando el micrófono a $15 \mathrm{~cm}$ y a un ángulo de $45^{\circ}$. El análisis acústico se realizó con el software PRAAT con el cual se obtuvo los valores para Jitter (perturbación de la frecuencia) y Shimmer (perturbación de la intensidad) del tramo medio del sonograma.

La evaluación fonoaudiológica, realizada por un solo examinador, incluyó además la graduación subjetiva de la disfonía, es decir, el grado general del desvío de la voz, la que podría darse en tres niveles: grado 1 (leve) para alteraciones vocales discretas, grado 2 (moderado) para alteraciones evidentes y grado 3 (severo) para alteraciones vocales intensas.

Luego de la evaluación inicial se comenzó la terapia vocal una vez por semana con una duración aproximada de 45 minutos. A cada uno de los pacientes se le aplicó un programa de actividades agrupadas en 7 unidades, las cuales se adaptaron a los distintos requerimientos que puede presentar un paciente dependiendo del grado y de las causas de la disfonía:

1. Relajación

2. Respiración

3. Técnica de resonancia

4. Suavización del ataque vocal

5. Tono vocal

6. Intensidad

7. Integración de parámetros vocales

Si bien el programa de intervención presentó variaciones en su aplicación, cada una de estas variaciones estaban orientadas a conseguir una voz estable, sin tensiones y de intensidad, tono y timbre adecuados, es decir, orientadas a que el paciente aprenda y aplique la técnica vocal en la voz hablada.

El término de la terapia se decretó una vez que el paciente logró los objetivos de cada unidad y después de haber ejercitado las condiciones ideales de producción de la voz hablada. La duración general del programa estaba condicionada por la asistencia, la ejercitación en el hogar y las condiciones de salud generales (resfríos, alergias, etc.), todos antecedentes registrados en la base de datos.

Los resultados de las evaluaciones iniciales y finales se compararon a través del análisis estadístico de T de Student con el apoyo del software SPSS.

\section{RESULTADOS}

En la evaluación inicial se registró para el índice Jitter un promedio de 1,09, una desviación típica de 0,86 , y un valor mínimos de 3,81 y un máximo de 0,29 . En la evaluación final el promedio de este indicador fue de 0,54, con una desviación típica de 
0,60, un valor mínimo de 0,18 y un valor máximo de 2,87. La diferencia existente entre ambos promedios fue de 0,55 , indicando una reducción de la perturbación de la frecuencia (Jitter) en el $50 \%$ (ver Tabla 1 y Figura 1).

El primer registro del índice Shimmer corresponde a 6,92 como promedio, con una desviación típica de 4,14, un valor mínimo de 1,30 y un máximo de 18,92. En el registro final se obtuvo un promedio de 3,43, una desviación típica de 1,34, un valor mínimo de 1,30 y un máximo de 5,88. La variación entre ambos promedios fue de 3,49 evidenciando una reducción de $50 \%$ en la perturbación de la intensidad (Shimmer) (ver Tabla 2 y Figura 2).

La aplicación de la prueba T de Student muestra una variación estadísticamente significativa entre la evaluación inicial para los índices Jitter y

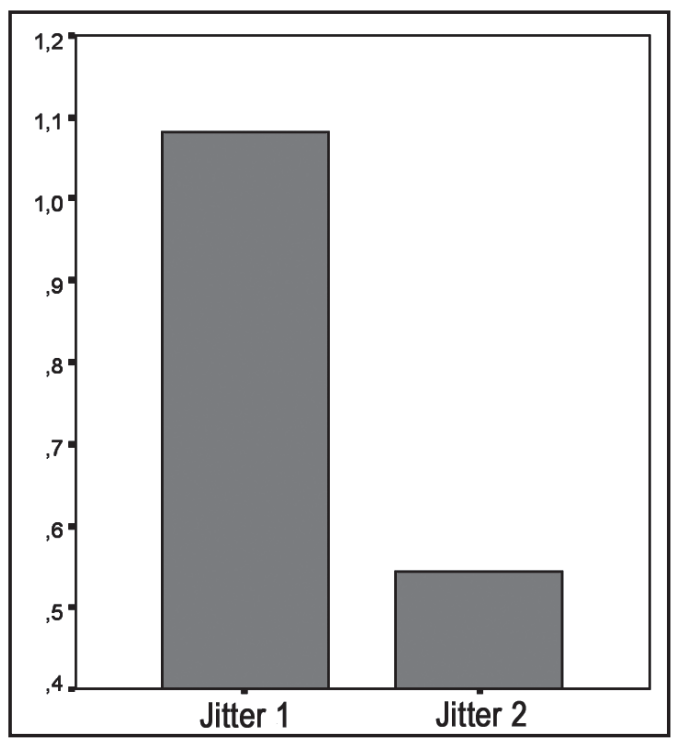

Figura 1. Comparación Jitter 10 inicial, y Jitter 20 final.
Shimmer, y la evaluación final de los mismos, pues el valor $p$ es inferior a 0,05 (ver Tabla 3).

El promedio del grado de disfonía resultante de la evaluación subjetiva inicial fue de 2 (moderado), registrándose pacientes con disfonía en grado 1 (leve) como valor mínimo y en grado 3 (severo) como máximo. Al término de la terapia se observó un promedio del grado disfonía de 1 (leve) siendo este mismo el valor máximo, mientras que el mínimo fue de 0 , es decir, ausencia de disfonía. La reducción del grado de disfonía observado fue del $50 \%$ ) (ver Tabla 4 y Figura 3 ).

Las características de los sujetos seleccionados para el presente estudio, fueron diversas en cuanto a edad, sexo y tipo de disfonía, por lo que los resultados de perturbación de Jitter y Shimmer caso a caso fueron también variados, en relación a estas condiciones. Esto se puede apreciar en las Tablas 1 y 2 al observar los valores mínimos y máximos. Sin embargo y teniendo esto en cuenta este escenario, es posible afirmar que del grupo total de los sujetos, el 93\% registró una disminución de la perturbación de la frecuencia fundamental, el $85 \%$ de los pacientes redujo la perturbación de la intensidad registrada en el análisis acústico. Por otro lado el $100 \%$ mejoró la cualidad de la voz representada por el grado de disfonía, aunque no todos los pacientes consiguieron una reducción total. Todas las variaciones registradas fueron estadísticamente significativas de acuerdo al análisis con la prueba T de Student del software SPSS (ver Tablas 3 y 5 ).

Las pruebas estadísticas aplicadas indican que la variación, o más bien la reducción de los índices Jitter y Shimmer, y del grado de disfonía fue independiente del sexo, de la edad y del tipo de disfonía. Tampoco se observaron relaciones significativas entre esta reducción y el tiempo de tratamiento, la asistencia a la terapia y la presencia de factores psicógenos como el estrés o depresión.

Tabla 1. Resultados medición de Jitter al inicio de la teriapia, posterapia y grado de variación

\begin{tabular}{|l|c|c|c|c|}
\hline Índice & Promedio & Desviación estándar & Valor mínimo & Valor máximo \\
\hline Jitter inicial & 1,09 & 0,86 & 0,29 & 3,81 \\
\hline Jitter final & 0,54 & 0,60 & 0,18 & 2,87 \\
\hline Variación Jitter inicial y final & $0,55=50 \%$ & & \\
\hline
\end{tabular}


Tabla 2. Resultados medición de Shimmer al inicio de la terapia y posterapia

\begin{tabular}{|l|c|c|c|c|}
\hline Índice & Promedio & Desviación estándar & Valor mínimo & Valor máximo \\
\hline Shimmer inicial & 6,92 & 4,14 & 1,30 & 18,92 \\
\hline Shimmer final & 3,43 & 1,34 & 1,30 & 5,88 \\
\hline Variación Shimmer inicial y final & $3,49=50 \%$ & & \\
\hline
\end{tabular}

\section{DISCUSIÓN}

La intervención fonoaudiológica en la disfonía se basa en principios subjetivos que giran en torno a sensaciones propioceptivas que cada paciente debe desarrollar para poder ejecutar concientemente cambios en la manera de producir la voz que lo lleva a obtener un mejor rendimiento vocal desde el punto de vista fisiológico y acústico. La aplicación de principios terapéuticos como el de la adecuada relajación, respiración e impostación de la voz resulta variable en cada caso, ya que se debe responder a las necesidades cognitivas y socioafectivas de cada sujeto, lo que lleva a interpretar la implementación terapéutica como un hacer dinámico, flexible y con un carácter subjetivo a la hora de evaluar los procedimientos realizados.

Como un intento de objetivizar y unificar criterios tanto de evaluación como de intervención, se ha incorporado a la clínica fonoaudiológica el uso de análisis acústico con softwares computacionales que han permitido generar perfiles acústicos de voces tanto normales como patológicas. En el contexto de las disfonías, las investigaciones realizadas establecen que los indicadores posibles

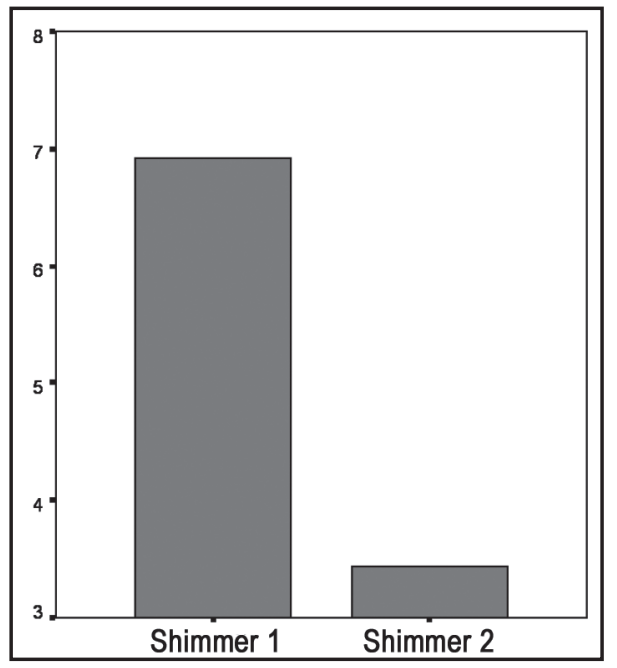

Figura 2. Comparación Shimmer 10 inicial y Shimmer 20 final.

de obtener van a ser siempre más altos en las disfonías en contraste con los registros de las voces normales. Sin embargo, falta claridad para determinar en qué momento exacto cierto valor es indicador de disfonía o cuánto es lo que éstos pueden 0 deben variar para determinar el término

Tabla 3. Resultados de la prueba T de Student proporcionado por el software Spss. Aquí se evidencian las diferencias significativas entre las evaluaciones iníciales y finales

\begin{tabular}{|c|c|c|c|c|c|c|c|c|}
\hline & \multicolumn{8}{|c|}{ Diferencias relacionadas } \\
\hline & \multirow[t]{2}{*}{ Media } & \multirow[t]{2}{*}{$\begin{array}{l}\text { Desviación } \\
\text { típ. }\end{array}$} & \multirow[t]{2}{*}{$\begin{array}{l}\text { Error típ. de } \\
\text { la media }\end{array}$} & \multicolumn{2}{|c|}{$\begin{array}{l}95 \% \text { Intervalo de confianza } \\
\text { para la diferencia }\end{array}$} & \multirow[t]{2}{*}{$t$} & \multirow[t]{2}{*}{$g l$} & \multirow{2}{*}{$\frac{\text { Sig. (bilateral) }}{\text { p-valor }}$} \\
\hline & & & & Inferior & Superior & & & \\
\hline $\begin{array}{ll}\text { Par } 1 & \text { JITTER1 } \\
& \text { JITTER2 }\end{array}$ & ,5404 & ,63636 & ,12247 & 2886 & ,7921 & 4,412 & 26 & ,000 \\
\hline $\begin{array}{rr}\text { Par } 2 & \text { SHIMER1 } \\
& \text { SHIMER2 }\end{array}$ & 3,4870 & 3,55933 & ,68499 & 2,0790 & 4,8951 & 5,091 & 26 &, 000 \\
\hline
\end{tabular}


Tabla 4. Grado de disfonía al inicio de la terapia y posterapia

\begin{tabular}{|l|c|c|c|}
\hline Indicador & Promedio & Valor mínimo & Valor máximo \\
\hline Grado de disfonía inicial & 2 & 1 & 3 \\
\hline Grado de disfonía final & 1 & 0 & 1 \\
\hline Variación del grado de disfonía inicial y final & $1=50 \%$ & \\
\hline
\end{tabular}

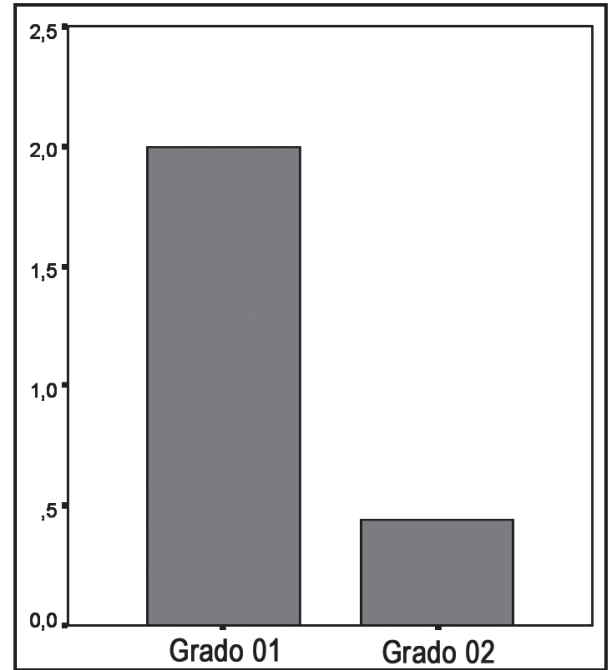

Figura 3. Comparación entre el grado de disfonía inicial y final.

de un proceso terapéutico. Las aplicaciones más usuales del análisis acústico en la terapia vocal guardan relación principalmente con la didáctica y el monitoreo continuo en conjunto con el paciente, y en investigación se ha utilizado para medir re- sultados en relación a procedimientos específicos, no así para el resultado global al usar una técnica vocal en el contexto del tratamiento de la disfonía.

En el presente trabajo, la reducción de las perturbaciones de la frecuencia y de la intensidad (Jitter y Shimmer) al finalizar la intervención evidencian objetivamente la evolución positiva de un paciente disfónico tras la terapia vocal. Sin embargo, al comparar estos resultados con otras investigaciones sobre los cambios pre y posterapia de los índices Jitter y Shimmer los resultados son bastante disímiles, por ejemplo, en el estudio realizado por Galarza y Pijoan en $2002^{10}$ no se observaron cambios significativos, excepto en aquellos pacientes con nódulos vocales. Mackenzie, Millar, Wilson, Sellars y Dearyen en $2001^{8}$ trabajaron con disfonías funcionales en donde no obtuvieron cambios luego de 14 sesiones. Por otra parte, Dejonckere, Bradley, Clemente, Cornut, CrevierBuchman, Friedrich G, Van de Heyning, Remacle y Woisard, en $2001^{14}$ y Vila-Rovira, Valero-García y González-Sanvisens en $2011^{15}$, sugieren que estos indicadores no son útiles en la valoración de la calidad vocal. Sin embargo, existen otros trabajos que indican lo contrario, como el de Piñero y Pire en $2002^{16}$ quienes reportaron mejorías en todos

Tabla 5. Resultados de la prueba estadística T de Student proporcionados por el programa Spss, que indican una diferencia significativa entre el grado de disfonía 10 inicial y el grado de disfonía 20 final

\begin{tabular}{|l|c|c|c|c|c|c|c|c|}
\hline & \multicolumn{6}{|c|}{ Diferencias relacionadas } \\
\hline & Media & $\begin{array}{c}\text { Desviación } \\
\text { típ. }\end{array}$ & $\begin{array}{c}\text { Error típ. de } \\
\text { la media }\end{array}$ & $\begin{array}{c}95 \% \text { Intervalo de confianza } \\
\text { para la diferencia }\end{array}$ & $\mathrm{t}$ & $\mathrm{gl}$ & $\begin{array}{c}\text { Sig. } \\
\text { (bilateral) }\end{array}$ \\
\hline & & & & Inferior & Superior & & & p-valor \\
\hline $\begin{array}{l}\text { Par 1 } \\
\text { Grado disfonía 1 } \\
\text { Grado disfonía 2 }\end{array}$ & 1,56 &, 641 &, 123 &, 123 & 1,30 & 12,619 & 26 &, 000 \\
\hline
\end{tabular}


Ios parámetros evaluados, incluidos los de Jitter y Shimmer y concluyen que el análisis acústico fue efectivo para evaluar la terapia de la voz ${ }^{16}$.

Otros trabajos más recientes, como el de Gutiérrez, del Prado y Asencio en 2012 ${ }^{17}$, muestran que luego de 10 sesiones se observan cambios perceptuales subjetivos en la calidad de voz, manifestada por una mejor percepción y una reducción de Jitter, aunque no así de Shimmer, siendo éste el índice menos sensible.

Las discrepancias descritas entre los resultados del presente trabajo y los resultados de otras investigaciones, puede deberse a diferencias metodológicas, vinculadas principalmente con el tiempo de tratamiento, el cual fue adaptado a las necesidades de cada paciente, lo que pudo haber potenciado un resultado mayormente positivo.

Lo anterior permite señalar la importancia de hacer investigaciones más profundas sobre la eficacia de la terapia vocal y las condiciones que son necesarias para que dicha terapia sea exitosa.

Por último, la independencia de la reducción del grado de disfonía revela la autonomía de la técnica vocal por sobre las variables observadas: edad, sexo, tiempo de tratamiento, tipo de disfonía, etc. En relación a esta última, se revela la utilidad de la terapia vocal independiente de las lesiones a nivel laríngeo de los pacientes de esta muestra, ya sea por parálisis de cuerda vocal, nódulos cordales o lesiones por reflujo faringolaríngeo, puesto que en todos los casos se observó reducción de los síntomas clínicos medidos. De esta manera es posible concluir que la disfonía, luego de la terapia vocal, debe reducirse en al menos $50 \%$ y que esta reducción perceptual es coherente con la variación de los índices acústicos Jitter y Shimmer, como medidas objetivas de la calidad vocal.

\section{CONCLUSIÓN}

Con el tratamiento propuesto la cuantificación objetiva de la evolución en la calidad de la voz de pacientes disfónicos a través de los índices de perturbación de la frecuencia fundamental (Jitter) e intensidad (Shimmer) obtenidos con el software de análisis acústico PRAAT reveló la reducción de estos índices en coherencia con el grado de disfonía obtenido de la evaluación cualitativa.
Los resultados obtenidos muestran la efectividad del tratamiento de la disfonía por parte del fonoaudiólogo cuando el paciente, comprometido con su propio proceso de aprendizaje, se somete a todas las etapas que implican la adquisición y el uso constante de una técnica vocal adecuada.

Aunque los índices Jitter y Shimmer, en el presente trabajo, se mostraron evidentemente sensibles ante la aplicación de terapia vocal, es necesario realizar más investigaciones, idealmente con muestras más amplias, para poder generalizar la premisa de que la evaluación objetiva de la evolución de la calidad de voz en una intervención terapéutica puede reflejarse en la variación de estos parámetros.

Del mismo modo siempre será necesario realizar un análisis acústico riguroso, completo y acabado para describir y explicar los fenómenos que ocurren en el tracto vocal que determinan la emisión y la calidad de la voz. Esto exige un alto rigor metodológico, para establecer las variables que más fomentan el éxito terapéutico.

Finalmente, la experiencia con los pacientes disfónicos del Hospital de La Serena presentada puede aportar en la continua búsqueda de la comprensión y explicación objetiva de los cambios obtenidos en la intervención terapéutica de la disfonía.

Esta investigación se realizó en el marco del Proyecto DIULS PF/07201 de la Dirección de Investigación de la Universidad de La Serena.

\section{BIBLIOGRAFÍA}

1. Jackson-Menaldi, Cristina. (2002). La Voz Patológica. Editorial Médica Panamericana.

2. Farías, Patricia G. (2007). Ejercicios para restaurar la función vocal, observaciones clínicas. Editorial Librería Akadia.

3. Belhlau \& Pontes (1989) citado por Farías, Patricia G. (2007). Ejercicios para restaurar la función vocal, observaciones clínicas. Editorial Librería Akadia.

4. Alonso Jesús, Ferrer Miguel, de León José, Travieso CarLOS. (2006) Cuantificación de la calidad de voz para la evaluación clínica por medio del análisis acústico. IV Jornadas en tecnologías del Habla, Zaragoza. 
5. Cecconello Luis. Valores de referencia de índices de perturbación a corto y largo plazo, medidas de ruido y F0 en niños. Revista de la Federación Argentina de Sociedades de Otorrinolaringología 2009; 16(2): 51-6.

6. Jackson-Menaldi Cristina. La Voz Normal. Editorial Médica Panamericana. 1992.

7. Boersma $P$ y Weenink D (2011). Praat: Doing phonetics by computer (Versión 5.2.21). http:// www.fon.hum.uva.nl/praat/

8. Mackenzie K, Millar A, Wilson Ja, Sellars C y DeaRY IJ. Is voice therapy an effective treatment for dysphonia? A ramdomized controlled trial. British Medical Journal 2001; 323: 1-6.

9. Hakkesteegt MM, Brocaar MP y Wieringa MH. The applicability of the Dysphonia Severity Index and the Voice Handicap Index in Evaluating Effects of Voice Therapy and Phonosurgery. Journal of Voice 2010; 24: 199-205.

10. Galarza I y Pijoan JI. El análisis acústico de voz en la rehabilitación de las disfonías. Revista de Logopedia, Foniatría y Audiología 2002; 22: 151-6.

11. Speyer R, Wieneke GH y Dejonckere PH. Documentation of progress in voice therapy: Perceptual, acoustic, and laryngostroboscopic findings pre therapy and post therapy. Journal of Voice 2004; 18: 325-40.

12. Quiñones Carmen. (1997). El cuidado de la voz, ejercicios prácticos. Editorial Escuela Española.
13. Tulon Carmen. (2000). La Voz: técnica vocal para la rehabilitación de la voz en las disfonías funcionales.

14. Dejonckere Ph, Bradley P, Clemente $P$, Cornut G, Crevier-Buchman L, Friedrich G, Van de Heyning P, Remacle M y Woisard V. A basic protocol for functional assessment of voice pathology, especially for investigating the efficacy of (phonosurgical) treatments and evaluating new assessment techniques. Guide line elaborated by the Committee on Phoniatrics of the European Laryngological Society (ELS). European Archives of Oto-rhinolaryngology 2001; 258: 77-82.

15. Vila-Rovira JM, Valero-García J y GonzálezSANVISENS L. Indicadores fonorrespiratorios de normalidad y patología en la clínica vocal. Revista de Investigación en Logopedia 2011; 1 : 33-55.

16. Piñero S, Pire B. (2002). Análisis acústico de la voz en pacientes con disfonía funcional, antes y después de la terapia. Servicio de Foniatría. Hospital Central "Antonio María Pineda". Barquisimeto. Marzo 1996 - febrero 1997. Boletín Médico de Posgrado. Vol XVIII No 1.

17. Gutiérrez A, del Prado M, Asensio CF. Eficacia de la reducción vocal en 10 sesiones clínicas. Revista de Investigación en Logopedia 2012; (2): 38-53. 